\title{
Historias de vida de mujeres militares en Colombia y Estados Unidos: un análisis reflexivo ${ }^{1}$
}

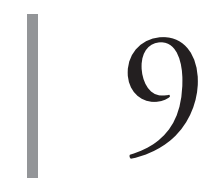

https://doi.org/10.21830/9789585318304.09

\author{
Natalia Pinzón Zambrano ${ }^{2}$ \\ Maria Alejandra Cardona Quintero ${ }^{3}$ \\ Edna Jackeline Latorre Rojas ${ }^{4}$ \\ Escuela Militar de Cadetes "General José María Córdova”
}

\section{Resumen}

El objetivo de este capítulo es conocer el papel de la mujer en el Ejército de Colombia y de Estados Unidos, a partir del relato de sus propias experiencias, con el fin de comparar y entender cómo ha sido su inclusión en cada uno de estos ejércitos. Las categorías centrales de estudio son la motivación para ingresar al ejército, las dificultades en combate, la importancia de la mujer en el combate y el desarraigo de la vida civil. Se aplicó el método analítico-deductivo de carácter fenomenológico. Mediante entrevistas semiestructuradas en una muestra no intencionada de diez mujeres se exploran cuestiones para entender cómo se han sentido a lo largo de la carrera, qué las motivó a ingresar a las Fuerzas Militares y cuáles son las dificultades que han sentido al ser mujeres militares. Finalmente, el estudio concluye con un análisis reflexivo de las experiencias de estas mujeres en la carrera de las armas.

Palabras clave: combate; enfoque de género; inclusión; mujer; mujer militar

1 Este capítulo presenta los resultados del proyecto de investigación "Atenea: caracterización sociodemográfica y su relación con la cultura de equidad de los integrantes de la Escuela Militar de Cadetes General José María Córdova. Segunda fase” del Grupo de Investigación en Ciencias Militares, de la Escuela Militar de Cadetes "General José María Córdova" (ESMIC), categorizado en B por Minciencias y con código de registro COL0082556. Los puntos de vista y los resultados de este artículo pertenecen a los autores y no reflejan necesariamente los de las instituciones participantes.

2 Subteniente del Ejército Nacional de Colombia. Profesional en Relaciones Internacionales (Escuela Militar de Cadetes "General José María Córdova”).

3 Subteniente del Ejército Nacional de Colombia. Abogada (Escuela Militar de Cadetes "General José María Córdova").

4 Magíster en Educación e Investigación Universitaria (Universidad Sergio Arboleda). Especialista en Educación e Investigación Universitaria (Universidad Sergio Arboleda). Psicóloga (Universidad Católica de Colombia). Directora del Observatorio Militar para la Igualdad entre Hombres y Mujeres. Investigadora del Grupo de Investigación en Ciencias Militares, de la Escuela Militar de Cadetes "General José María Córdova". Orcid: https://orcid.org/0000-0002-0301-9036 - Contacto: edna.latorre@esmic.edu.co 


\section{Introducción}

El propósito de este escrito es acercarse a un análisis reflexivo de las experiencias relacionadas con el enfoque de inclusión y género que han tenido los ejércitos de Colombia y de Estados Unidos. Para tal fin se explora el proceso de incorporación de la mujer en las Fuerzas Armadas, las condiciones que les permiten estar en posiciones de combate (como es el caso de las mujeres militares en EE. UU.) y las situaciones que han afrontado al ocupar estas posiciones. En consecuencia, se plantea un recorrido teórico por algunos estudios de la inclusión mujeres militares estadounidenses y la evolución de esta inclusión hasta la actualidad, como supuestos que fundamentan el estudio. Cabe destacar que se revisan los modelos adoptados por el ejército de Estados Unidos en su sistema militar en cuanto a la inclusión y la igualdad de las mujeres en las filas. Otro aspecto que fundamenta el presente estudio es la adopción de la teoría de identidad social de Henri Tajfel (1978) como cimiento para acercarse a una concepción del enfoque de género de la investigación.

Es importante comprender, con respecto a la participación en las guerras, que es la figura masculina la que ha estado fuertemente asociada a estas desde hace muchos años, mientras que la figura femenina ha estado asociada a la paz. No obstante, la intervención de la mujer en la guerra se extiende a más de cuatro mil años en el pasado a través de un gran número de culturas, ya sea tomando las armas o como auxiliares de los guerreros, arrojando piedras (como las iberas o las guerras del Peloponeso). La guerra siempre ha sido objeto de preocupación y posicionamiento, tanto individual como colectivo, para las mujeres en todas las épocas históricas (Gallardo, 2012). Aunque pasaran guerras y los conflictos siguieran, la participación de la mujer en combate no estaba permitida, por tanto lo hacían encubiertas como hombres, como fue caso de la estadounidense Deborah Sampson, que bajo el nombre de Robert Shurtliff, luchó en el Ejército Continental durante la guerra de Independencia, fue herida en 1782 y dada de baja con honor en West Point, Nueva York en 1783 (Gallardo, 2012).

Durante la Primera Guerra Mundial, en 1914, a causa de la gran cantidad de tropas movilizadas, la industria, motor de la guerra, requería trabajadoras, es por esto que se pensó en el personal femenino, por lo cual la mano de obra femenina supuso el $40 \%$ de la producción y gran parte de la industria armamentista. También, fueron empleadas en unidades de inteligencia con el fin de engañar a los hombres enemigos, como el caso de Margaretha Greertruida Zelle, más conocida como Mata Hari, una famosa bailarina y actriz condenada a muerte por espionaje y ejecutada por fusilamiento en 1917 (González, 2005) 
En Colombia, las mujeres en el campo militar iniciaron con labores de enfermería. Se resalta el caso de Clara Elisa Narváez Arteaga, quien participó en la guerra de Colombia contra Perú en 1932, cumpliendo labores de enfermera de combate. Dada la escasez de enfermeras, fue la única mujer que acompañó las tropas colombianas, por lo cual abandonó su familia y su pueblo para proteger a su país.

Casos como los anteriores fueron llenando la historia de la incursión de las mujeres en los ejércitos, quienes desempeñaron un papel importante dentro de la guerra. En el 2000, las Naciones Unidas adoptaron la Resolución 1325 del Consejo de Seguridad, en la que se buscaba poner fin a la desigualdad histórica de la participación de hombres y mujeres en el momento de asegurar la paz de sus países. Con dicha Resolución, se estableció un marco jurídico y político que exigía a las partes del conflicto tener en cuenta a la mujer como mediadora y negociadora no solo por sus valores y compromiso para evitar la violencia, sino por su derecho en la normativa internacional (Gallardo, 2012).

Durante el 2000, solo cinco países de la OTAN (Organización del Tratado del Atlántico Norte) disponían de políticas específicas para la participación de las mujeres en las Fuerzas Armadas. En el 2007, la OTAN se propuso poner en práctica dicha Resolución para ampliar el papel de la mujer en las operaciones de Naciones Unidas. Todo esto quedó plasmado en el informe CWINF Guidance for NATO Gender Mainstreaming, y en el 2013, estas políticas ya estaban en 28 países. Aunque los países miembros ofrecieron igualdad de oportunidades a las mujeres, quedaron ciertas posiciones limitadas, principalmente posiciones de combate y algunas flotillas de submarinos (Caicedo et al., 2017).

\section{Marco teórico}

\section{La identidad como explicación de la construcción social del papel de la mujer}

El término identidad social es resultado de un proceso largo de investigación que ha cambiado la estructura de los sistemas y ha generado transformaciones (proceso adaptativo) y mutaciones (alteración cualitativa). En este orden de ideas, la identidad social ha llegado a orientarse por un conjunto de códigos y reglas, expuestas por Devereux (2014), como exigencias de cooperación y solidaridad. Como resultado se tiene una continuidad de relaciones sociales que trasciende más escenarios. El surgimiento de la identidad, en sus diferentes dimensiones, presupone una continuidad de las relaciones sociales en la vida del individuo, es decir, un mundo común que el individuo comparte, ya no solo con sus interlocutores 
próximos en las redes de la sociabilidad cotidiana, sino también con otros individuos más lejanos, desconocidos y anónimos. Dicho de otro modo, a la relación Ego-Alter, que constituye la condición elemental de emergencia de la identidad, se añade un tercer término: la estructura condicionante del mundo social más amplio (Herrera Gómez, 2000).

La identidad está determinada por las percepciones e imaginarios sociales de los individuos cuando consideran que hacen parte de un grupo, por lo tanto, cada ser humano cree que pertenece a una categoría social, por ejemplo, su género, y esta creencia afecta directamente todo su desarrollo personal. Para Tajfel, "los individuos percibirían la sociedad compuesta por multitud de categorías sociales y tendrían conciencia de su pertenencia a alguna(s) de esa(s) categoría(s)" (Canto \& Moral, 2005, p. 4).

De acuerdo con lo anterior, el desarrollo personal del ser humano depende de la categorización en diferentes dominios: la apariencia física, el comportamiento social, el desempeño académico y la influencia de las relaciones familiares, entre otros. De esta manera, los individuos crean una serie de estereotipos según caracterizaciones que lo hacen poseer rasgos de orden social y que les permiten pertenecer a un grupo o categoría con la que se sienten representados. Un claro ejemplo es la categorización del género: cuando el sistema de creencias es masculino, se determina una persona ruda o con gusto por la cerveza; en cambio, si se categoriza en el género femenino, se supone que la persona debe ser delicada y con gusto por ir de compras.

Pero no solo la identidad social constituye la personalidad del individuo, también se encuentra un componente personal, que en la teoría de la identidad social (TIS) se conoce como identidad personal. Esta identificación hace referencia a los aspectos individuales determinados por la genética y las experiencias propias: "identidad social se refiere a un sentimiento de similitud con otros, la identidad personal se refiere a otro sentimiento que lo diferencia de esos otros" (Canto \& Moral, 2005, p. 4).

En el momento en que se enfrentan la identidad social y la identidad personal el individuo inicia un proceso que TIS conoce como "sesgos cognitivos básicos", es decir, los pensamientos que las personas deben trabajar para poder crear una identidad propia y única. Entre estos sesgos están el favoritismo endogrupal y la homogeneidad exogrupal (Fernández \& Latorre, 2018)

La homogeneidad exogrupal es entendida como la creencia de que todos los miembros de un grupo externo a la persona son muy parecidos e incluso iguales entre sí. Esto produce los estereotipos de cada caracterización social, cuya homogenización se basa comúnmente en la raza, la religión, la nacionalidad, la especialización universitaria, la edad y el sexo, entre otros. Por lo tanto, los anteriores aspectos 
afectan la "legitimación social" de los roles del género masculino y femenino en la sociedad (Kimble et al., 2002).

Ahora bien, para hablar de equidad de género es importante tener en cuenta que este concepto ha sido estudiado y definido por varios intelectuales, como Brailovsky et al. (2001), Palomar Verea (2005) y Antolín (2003). Así, el género incide en el accionar de toda persona, mediante la asignación social de los roles dependiendo de su sexo, lo que se irá afianzando en las interacciones sociales; además, para la construcción de identidad de género se tendrá en cuenta una serie de reglas y roles asignados a cada persona (Brown \& Soto, 2007).

El panorama para Colombia en cuanto a equidad de género es bastante tardío, a pesar de haber contado desde el siglo XIX con grandes lideresas, pues fue hasta los ańos ochenta que, con la Ley 51 de 1981 sobre la eliminación de todas las formas de discriminación hacia la mujer, se abrió el paso a una era más imparcial. Aun cuando se establece una era más equitativa permanecen estereotipos de género muy arraigados en el país. Algunos estereotipos de género asociados a la mujer son estos: sumisa, emocional, tranquila, limpia, ordenada y artística. Con respecto a los hombres los estereotipos son los siguientes: agresivo, ruidoso, desordenado, atlético, orientado a la ciencia y a la matemática.

Los hombres y las mujeres no están imposibilitados de realizar ninguna actividad por el solo hecho de pertenecer a uno de estos géneros. Las diferencias entre ambos no son necesariamente obstáculos para incursionar en actividades tradicionalmente masculinas o femeninas: el trabajo y la voluntad de realización pueden superar cualquier dificultad (Brailovsky et al., 2001).

En conclusión, la equidad aumenta a partir de una identidad social más abierta y menos sesgada en la forma en que los demás perciben al individuo. Esa construcción del yo que se quiere ofrecer a los actores sociales va a tener relevancia en las relaciones sociales y los diferentes campos laborales. Los hombres, entonces, tienen que aceptar la figura de una mujer empoderada, capaz de alcanzar grandes logros, y dejar atrás el temor o la impotencia de ver a alguien del sexo femenino teniendo los mismos o mejores logros.

\section{Estados Unidos: punto de referencia en la inclusión de la mujer en el Ejército}

Los referentes teóricos que se presentan a continuación hacen parte de estudios realizados en cuanto a la caracterización sociodemográfica y la integración de las mujeres en el ejército de los Estados Unidos. Es importante aclarar que para el presente estudio los avances que ha tenido el ejército estadounidense en cuanto a 
la incorporación de las mujeres a las tropas resalta procesos que pueden ser incorporados en el ejército colombiano, debido a sus resultados y al reconocimiento de los Estados Unidos como el país potencia en cuanto a asuntos militares a nivel mundial. Además, la amplia experiencia de este país en asuntos de seguridad, desde su participación en la Primera y Segunda Guerra Mundial y en la guerra del Golfo Pérsico, lo posicionan como un referente en cuanto a las buenas prácticas de integración de la mujer en asuntos de seguridad y defensa.

Dentro del análisis de estudios realizados se destaca el de Asch et al. (2016). En su obra se desarrolla un estudio en dos fases para abordar la brecha en la información cuantitativa sobre las diferencias en la progresión de la carrera de oficiales según el sexo y los factores exógenos que explican estas diferencias. Otro trabajo destacado es el realizado por Szayna et al. (2016), en el que se evalúan los desafíos potenciales para la integración de las mujeres en las Special Operations Forces (SOF), obra que muestra cómo se validan los estándares ocupacionales SOF para las posiciones controladas por el Comando de Operaciones Especiales de EE. UU. El informe resume la historia de la integración de las mujeres en las fuerzas armadas de los EE.UU., revisa el estado actual del conocimiento sobre la cohesión en unidades pequeñas y analiza la aplicación de estándares de género neutrales a las SOF. Además, identifica estándares profesionales ampliamente acordados para la validación de ocupaciones físicamente exigentes y ayuda a los componentes del servicio SOF con la aplicación de estos estándares. El informe también analiza los datos primarios, una encuesta al personal de las integrantes de las SOF y una serie de discusiones de grupos focales sobre los posibles desafíos para la integración de las mujeres. El informe presenta recomendaciones sobre el proceso de implementación de la integración de las mujeres en SOF.

Finalmente, el trabajo de Schaefer et al. (2015) desarrolla una investigación para el Cuerpo de Marines de los EE. UU. sobre cuatro tareas: 1) revisar el literatura sobre la integración de mujeres en combate terrestre y otras ocupaciones físicamente exigentes, 2) entrevistar representantes de organizaciones que han integrado a las mujeres en ocupaciones físicamente exigentes, 3) estimar los costos de las potenciales iniciativas para promover el éxito integración de género y 4) desarrollar un enfoque para el monitoreo implementación de la integración de género en la infantería. Las preguntas de investigación fueron las siguientes: ¿Qué problemas pueden surgir si las mujeres están integradas en la Infantería del Cuerpo de Marines? ¿Qué esfuerzos han sido exitosos al abordar estos problemas en el pasado? ¿Qué costos potenciales son probablemente asociados con la integración del género? Como resultado presentan un resumen histórico de la integración de 
las mujeres en el Ejército de los EE. UU. Además, exploran la importancia de la cohesión y la influencia. El informe culmina con un resumen de los esfuerzos de monitoreo previos, las estrategias para esta actividad y las recomendaciones para la implementación de la equidad en el cuerpo de Marines.

A partir del recuento anterior se piensa en los aspectos que debe contemplar la integración de las mujeres en las fuerzas armadas. Al respecto, Echevarría (1990) refiere que existen cuatro factores que actúan de forma conjunta para permitir la integración de la mujer en las fuerzas armadas de los países occidentales. El primero sería el factor político, que permite el reconocimiento de los derechos de la mujer y la igualdad frente a los demás miembros. El segundo es el factor laboral, que permite el trabajo sin discriminación. El tercer factor es el sociológico, que evite el encasillamiento en los roles tradicionales por sexo y que haga posible mayor equidad en la delegación de funciones y tareas. Por último, está el factor militar, que proporciona mayor profesionalización al personal de oficiales incorporado sin importar el género.

Cuando se analiza el marco legal de los derechos en Estados Unidos, se encuentra que los factores nombrados están relacionados específicamente en el título 2 de la Ley de Derechos Civiles de 1964 de Estados Unidos. Dicha ley incluye a las mujeres en los estatutos antidiscriminatorios, ya que antes no eran asignadas a todos los puestos que requería la Fuerza, en especial, aquellos en los que estaban expuestas al combate directo. Así, estaba cerrado el acceso, por ejemplo, a la infantería, al arma acorazada, a los zapadores, a operaciones especiales, a la artillería antiaérea (corto alcance), a la artillería de campaña y a ser piloto de helicóptero de ataque, funciones que se veían restringidas no por la ley, sino por una política general.

Lo anterior legitimó el papel de las mujeres en el Ejército estadounidense y permitió que se asimilara su presencia en el sector defensa del país. A esto se sumó la promoción de la igualdad en diferentes conferencias sobre relaciones humanas y conductas sexuales, entre otras. Un claro ejemplo de ello es que en 1976 ingresaron las primeras cadetes en West Point. Era un total de 119 alumnas, que estudiaron por cuatro años, de las cuales se graduaron 64 (el 10 a $12 \%$ ). Al principio ellas no recibían en su totalidad los mismos entrenamientos que los cadetes, por ejemplo, en vez de tomar clases de boxeo, ellas entrenaban defensa personal (González, 2005).

Dave Philipps y Thomas Gibbons, corresponsales nacionales que cubren notas sobre veteranos y militares en Estados Unidos, afirman al respecto: "Otros creerán que es un paso atrás para otras ramas militares, lo que sí es cierto es que es un hito para la infantería de Marina, por su resistencia al integrar mujeres a sus fuerzas de 
combate" (Philipps, 2017). Por otra parte, en el entrenamiento de infantería del Ejército norteamericano, hombres y mujeres han caminado por el bosque en escuadrones mixtos durante dos años, pero para los infantes de marina "unir hombres y mujeres distraería a los reclutas y quizás intimidará a las infantes de marina”. No obstante, no todos piensan así, pues, según un oficial de la Marina que se negó a ser nombrado porque no se le permitía hablar públicamente, este avance por integrar a la mujer con los hombres se debió a la falta de reclutas en el tiempo de entrenamiento de invierno.

Últimamente en el Ejército de Estados Unidos se han evidenciado intenciones de inclusión de las mujeres en cargos anteriormente dispuestos para hombres, pero frente a esto se han creado barreras y dualidades, con afirmaciones sobre la rebaja en estándares físicos que significaría la presencia de las mujeres en la Marina. Por ejemplo, la teniente coronel retirada Kate Germano, quien dirigió el batallón de mujeres segregadas en la Isla Parris de 2014 a 2015, fue expulsada luego de criticar cómo se capacitaron las reclutas, porque según ella, se les dieron estándares de entrenamiento más bajos y frecuentemente eran objeto de burla por parte de los hombres, quienes abandonaban el campo de entrenamiento. Respecto a su opinión, los funcionarios de la Isla Parris y el Pentágono se negaron a dar explicaciones (Philipps, 2019). Aun así, sus niveles de inclusión han crecido significativamente.

Debido a esta política de inclusión en puestos de combate, las militares norteamericanas han podido participar en roles anteriormente imposibles para ellas. Por ejemplo, dos mujeres han marcado un hito en la historia al convertirse en las dos primeras soldados en superar las pruebas de la Escuela de los Ranger dentro del Regimiento de Infantería Ligera. Ellas, junto con 94 hombres, superaron los 62 días del curso de liderazgo, que adoctrina a los participantes en cómo combatir la fatiga, el hambre y el estrés para dirigir a los soldados durante pequeñas operaciones en la unidad de combate.

Este curso desarrollado en Fort Benning, Georgia,

Consistía en difíciles entrenamientos en bosques, terrenos montañosos y pantanos de Florida, además exigía entrenamiento físico, como 49 flexiones de brazo, 59 abdominales, una carrea de cinco millas en 40 minutos y 6 sentadillas; una prueba de natación, de navegación, una marcha a pie de 12 millas en tres horas, distintos cursos de obstáculos, cuatro días de entrenamiento en la montańa, saltos en paracaídas; asaltos aéreos en helicópteros, múltiples movimientos en barco; y 27 días de patrullas de combate simuladas. (Dos mujeres hacen historia militar en EE. UU. al convertirse en las primeras 'Ranger', 2015) 
De acuerdo con lo anterior, el Ejército busca que hombres y mujeres carguen con los mismos morrales, arrojen las mismas granadas y porten las mismas ametralladoras. Igualmente buscan restar importancia a los nuevos soldados de infantería femenina, para que no se les vea como desiguales: en el curso participan desde atletas de preparatoria hasta una madre soltera con título culinario.

Las nueve compañías de infantería están integradas por escuadrones mixtos, se preparan desde que amanece hasta que anochece, duermen en habitaciones separadas por género y en literas de metal. Para que poderse graduar deben cumplir las siguientes pruebas: lanzar granadas a 35 metros, arrastrar 15 metros un maniquí de entrenamiento de 121,6 kilos y completar una marcha de 19,3 kilómetros cargando 30,8 kilos.

Es de resaltar que las mujeres norteamericanas han hecho un excelente trabajo no solo en los cursos militares, sino también en la guerra, puesto que se ha visto su participación directamente en enfrentamientos. Aunque no pertenezcan propiamente a tropas de combate, sí han desarrollado estas labores. Un claro ejemplo de esto es en Afganistán e Iraq, donde a casi 14.000 mujeres se les otorgó la insignia de Acción en Combate; allí fue completamente normal que la mayoría de los hombres que lideran el Ejército hubiera prestado servicio con mujeres. Muchos de ellos resaltan el desempeño de estas militares, por ejemplo, el mayor general Jeffrey Snow, quien afirma: "Ahora tenemos una generación que solo quiere cumplir la misión y tener a las personas más talentosas para hacerlo" (Philipps, 2017).

\section{Método}

El enfoque utilizado para el presente trabajo fue cualitativo, dentro del cual se buscó la interpretación, uso y recolección de información empírica, con el fin de revisar los momentos habituales desde la perspectiva de quienes están continuamente interpretándose y definiéndose en diferentes situaciones (Taylor \& Bogdan, 1998). A partir de esta información, se quiere visualizar qué piensa y qué hace en su diario vivir la población en estudio, en este caso, las mujeres estadounidenses y colombianas que se desempeñan como militares, para identificar las situaciones que viven en el área de combate o en su lugar de trabajo.

El método es fenomenológico y trascurre en tres etapas. La primera es la descripción, que se refiere al aporte teórico y conceptual del enfoque de género y su relación con el Ejército de los Estados Unidos. También hace referencia a la elección de la técnica de recolección de datos y el protocolo para la descripción de los hallazgos. La segunda etapa es la estructuración, que consiste en el alistamiento de 
las entrevistas y en determinar las características poblacionales de las once mujeres militares de Colombia y de Estados Unidos (dos y diez, respectivamente). Ellas de manera voluntaria fueron entrevistadas bajo el modelo de un muestreo no probabilístico en el que no se busca generalizar los hallazgos, sino comprender el papel que tiene la mujer en el Ejército. El instrumento de recolección de datos fue la entrevista semidirigida, construida con el fin de indagar sobre las experiencias a lo largo de su carrera profesional, los motivos que las llevan a ingresar a las Fuerzas Militares y las diferentes situaciones que viven a diario, de acuerdo con las categorías centrales del estudio (tabla 1). La tercera etapa es la discusión de los hallazgos.

Tabla 1. Cuadro de categorías centrales del estudio

\begin{tabular}{ll}
\hline \multicolumn{1}{c}{ Categoría } & \multicolumn{1}{c}{ Definición operacional } \\
\hline \multirow{2}{*}{ Motivación para ingresar al Ejército } & $\begin{array}{l}\text { Entendida como los impulsos que llevan a una persona a } \\
\text { escoger como proyecto profesional ser miembro del Ejército } \\
\text { Nacional. }\end{array}$ \\
& $\begin{array}{l}\text { Entendida como las situaciones difíciles de una mujer en } \\
\text { combate. }\end{array}$ \\
Dificultades en combate & $\begin{array}{l}\text { Entendida como el valor que tienen las habilidades y } \\
\text { destrezas de una mujer en el campo de combate. }\end{array}$ \\
Eesarraigo de la vida civil & $\begin{array}{l}\text { Entendido como los aspectos que añora o extraña de su vida } \\
\text { civil al ingresar al Ejército. }\end{array}$ \\
\hline
\end{tabular}

Fuente: Elaboración propia

Las entrevistas se hicieron de manera virtual y se dividieron en dos partes. En la primera se recolectaron datos sociodemográficos (edad, grado, lugar de procedencia, tiempo en la fuerza, lugar y área de trabajo). En la segunda, a partir de diez preguntas, se indagó sobre las categorías mencionadas anteriormente, en las cuales las participantes podían responder de manera abierta lo que se les preguntaba respecto a sus experiencias como mujeres militares.

El análisis de la información se hizo a partir del reconocimiento semántico de palabras como vocación militar, influencia familiar, cambios físicos, respeto, confianza, habilidades, familia y dependencia entre otras. De esta manera, se trianguló la información para poder adoptar diferentes perspectivas y dar respuesta a la problemática central del estudio. 


\section{Resultados}

Los resultados describen las experiencias que han tenido algunas mujeres militares del Ejército de Estados Unidos y del Ejército de Colombia. De igual manera, se presenta la triangulación hecha entre las categorías de estudio, las entrevistas de las mujeres militares estadounidenses y las entrevistas de las mujeres militares colombianas. Es importante anotar que las descripciones se hacen en torno a las cuatro categorías centrales que se proponen, a saber, 1) motivación al ingresar al ejército, 2) dificultades en combate, 3) importancia de la mujer en combate y 4) desarraigo de la vida civil.

\section{Experiencias de las mujeres militares del Ejército de Estados Unidos}

Los hallazgos dentro de esta exploración se hicieron a partir de las experiencias de vida de dos mujeres de Estados Unidos: una teniente coronel de 46 años, abogada y asesora jurídica, con trece años en la Fuerza, nacida en Minnesota; y una oficial técnica de rango CW3 de 40 años de edad, gerente de Proyectos y Operaciones de la Oficina Legal, con 18 años y medio en la Fuerza, nacida en New Jersey. Ellas se reconocerán como entrevistada 1 y 2 respectivamente. Las entrevistas tuvieron lugar de manera virtual a través de un formulario semiestructurado que indagaba sobre sus experiencias y percepciones como mujeres militares a partir de doce preguntas. $\mathrm{Al}$ respecto, los resultados encontrados fueron los siguientes:

Con respecto a las motivaciones para ingresar al Ejército, las entrevistadas manifiestan un impulso de desarrollo profesional que permitía la consecución de una carrera con beneficios salariales más fijos que cualquier otra. Esto se observa en la respuesta de la entrevistada 2: "Me motiva el dinero para la universidad y la opción de carrera"; sin embargo, no solo el empleo estable es una motivación. Llama la atención la respuesta de la entrevistada 1, quien resalta la importancia del servicio a la comunidad y la preocupación por los conflictos internacionales que desatan los hechos de guerra: "Quería servir a mi país. Me inspiró mucho el hecho de que había militares prestando servicio después de los ataques del 11 de septiembre del 2001, y en la invasión de Iraq en 2003". También ambas entrevistadas dan un valor motivador a las relaciones que se generan dentro de su mismo país y fuera de él: 
Tener la oportunidad de intercambiar ideas y experiencias con militares de otras culturas. Claro que me ha encantado poder tener intercambios con nuestros socios en Latinoamérica, dado mi interés en la región. Creo que todos podemos crecer como seres humanos y como militares, entre más nos conocemos y nos entendemos. (Entrevistada 1)

Claramente se observa la incidencia del sistema militar internacional en asuntos regionales y cómo desde la perspectiva de una mujer esta es una oportunidad de construcción de lazos que propician intercambio de experiencias para el fortalecimiento del sistema internacional.

En cuanto a la motivación por fortalecimiento de relaciones internas, es evidente la vocación de servicio a la comunidad a través de la labor que se desempeña como mujer militar cuando la entrevistada 2 afirma que siente satisfacción al "servir a las personas sabiendo que soy directamente responsable de su capacitación, sus evaluaciones, sus premios y de proporcionar elementos y recursos para ayudar a realizar sus tareas diarias".

Los hallazgos en cuanto a las dificultades en combate dejan ver que indudablemente las mujeres militares estadounidenses no se quedan en su país, por lo tanto, sus experiencias relatan los hechos que viven cuando son enviadas a otros países. Las dos entrevistadas comparten su experiencia en Iraq y cuentan que lo más difícil para ellas fue "balancear la vida militar y la vida familiar" (entrevistada 1), así como el desapego de sus hijos: "Dejar a mi hijo de 14 meses cuando me enviaron a Iraq" (entrevistada 2). Otro factor que se relata dentro de las situaciones difíciles en combate es el mismo ejercicio de liderazgo de la mujer, que la lleva a construir relaciones de confianza con su equipo. Al respecto, la entrevistada 1 afirmó: "Ganar la confianza de los hombres y los comandantes y convencerlos de que todos estamos en el mismo equipo y de que las mujeres no somos una amenaza para ellos en el campo de batalla es la primera dificultad con la que una mujer se encuentra”.

Otra dificultad en combate tiene que ver con las condiciones para garantizar la higiene personal:

Hay momentos en que una unidad está en misiones durante semanas o meses. Las toallitas para bebés no proporcionarán la limpieza que necesita una mujer. Un baño "de pájaro" no interrumpirá el periodo [...]. Las mujeres necesitan equiparse con kits especiales de higiene. Los hombres reciben kits de afeitado. (Entrevistada 2)

La siguiente exploración se hace en la categoría de importancia de la mujer en combate. Se reconoce el valor de la mujer como comandante, involucrada en el proceso de toma de decisiones y ejerciendo un papel de líder, desde el cual aporta 
al Ejército una perspectiva diferente que, a la vez, implica la puesta en escena de habilidades con las que cuentan las mujeres. Esto se observa cuando la entrevistada 1 afirma: "Además, en ambos casos hay que reconocer que las mujeres traemos una perspectiva y habilidades distintas; y creo que esto es bueno". Al respecto, la entrevistada 2 afirma:

La perspectiva de una mujer es única. Además, las mujeres han estado sirviendo en combate desde el principio de los tiempos. Nos hemos disfrazado para servir. Hemos servido en muchas otras compañías, pero es importante que las mujeres sirvan como comandantes de combate para que sean ellas quienes tomen las decisiones.

Estas respuestas demuestran que las entrevistadas ven en ellas habilidades y destrezas que fortalecen su labor como mujer militar. De manera específica reconocen que ellas no solo pueden ejercer una gran labor, sino que también pueden llegar a comandar una unidad cuando el servicio lo requiera.

Por último, se indaga sobre la cuarta categoría, desarraigo de la vida civil, con la pregunta ¿qué aspectos extrańaban de su vida civil? Frente a este interrogante, las entrevistadas centraron sus respuestas en la estabilidad que da ser civil en la vida cotidiana: "Extraño la estabilidad de la vida diaria en el lado civil; no hay muchas emergencias y todo va más o menos bien" (entrevistada 1). "Normalmente el Ejército nos exige que nos mudemos cada tres años, así que la vida militar puede no ser estable cuando eres madre" (entrevistada 2). El comentario final muestra una inclinación hacia la satisfacción de una identificación social de género, la de "mujer madre", que aun en países del primer mundo se vive como algo importante para la mujer.

\section{Experiencias de las mujeres militares del Ejército de Colombia}

Las mujeres militares entrevistadas fueron ocho: dos subtenientes recién graduadas de la Escuela Militar de Cadetes "General José María Córdova" (en adelante, ESMIC) (entrevistadas 1 y 2), dos tenientes que se desempeñan como comandantes del personal femenino de la ESMIC (entrevistadas 3 y 4), una mayor que se desempeńa como jefe del Departamento de Psicología de la ESMIC (entrevistada 5), dos capitanes del Ejército Nacional de Colombia (entrevistadas 6 y 7), y una teniente coronel del Departamento de Personal (CEDE-1) del Ejército (entrevistada 8). 
La exploración inicia en la categoría de motivación al ingresar al Ejército. Las entrevistadas manifiestan su vocación por el servicio como factor importante para tomar la decisión de incorporarse al Ejército: "sentir la necesidad de servir a la población” (entrevistada 3), "mi familia y la colaboración a la población civil que hace el Ejército" (entrevistada 4) y "poder servir a la sociedad" (entrevistada 6).

En la categoría de dificultades en combate es importante reconocer que en Colombia se vive un conflicto interno desde hace más de 50 años. La guerra irregular hace de las condiciones políticas y sociales un factor que demanda un Ejército robusto y fortalecido para poder enfrentar los componentes de este tipo de guerra. El entrenamiento militar, entonces, constituye un elemento clave para el ejercicio de las labores de tropa. En Colombia, los soldados en su ciclo de operaciones, descanso y entrenamiento (en adelante, CODE) duran aproximadamente cinco meses. Hoy por hoy, en las diferentes variaciones del conflicto armando, este tiempo puede ser hasta de siete meses. Cabe decir que ellos tienen sus provisiones y cada quince días los abastecen. Normalmente, por las dificultades del terreno y situación operacional, hay lugares a los que no se pueden desplazar con vehículos debido a la presencia de grupos armados organizados (GAO). Al respecto la entrevistada 8 manifiesta:

Una mujer que está en la capacidad de ir al área tiene que ser una mujer excelente físicamente, que no le importen las incomodidades de la selva, como no bañarse todos los días, soportar cólicos, mareos, sabiendo que las bases no están cerca de su ubicación y estar segura que sus hombres la van a respetar a la hora de impartir una orden, demostrando ejemplo y liderazgo.

Además, una mujer combatiente debe cargar entre 45 y $70 \mathrm{~kg}$ y caminar marchas de 5 a $12 \mathrm{~km}$, con alturas de 200 a 1.800 metros con las características particulares del territorio colombiano. Debe estar interna con soldados que están prestando servicio militar obligatorio o soldados profesionales, quienes no están acostumbrados a ver mujeres militares con mando de tropa.

Otra dificultad que manifiestan tener las entrevistadas es la que tiene que ver con las condiciones de salubridad e higiene: "lo más difícil para una mujer en el combate van a ser las condiciones higiénicas, el esfuerzo físico y el mando con los soldados" (entrevistada 5); "la menstruación" (entrevistada 7). La entrevistada 3 dice que "la adaptación a la vida militar", mientras que la respuesta de la entrevistada 4 es "el acoso sexual y laboral".

Al explorar la categoría de importancia de la mujer en el combate, los hallazgos muestran respuestas sexistas y estereotipadas, en las que las entrevistadas piensan que 
una mujer en al área podría permitir más acceso a la población civil que un hombre y, visitando hogares vulnerables, prestando servicios médicos, dialogando con las familias de comunidades que muchas veces no son afectos al Ejército, recolectando información de la presencia del enemigo, y ayudar así al desarrollo de operaciones. (Entrevistada 2)

En contraste, la entrevistada 5 responde:

No considero que sea esencial, ya que las tareas que se efectúan en el área de operaciones son muy complejas y, si bien a una mujer no le queda imposible efectuarlas, le va a costar mucho trabajo adaptarse a un entorno al que no está acostumbrada, donde requiere un esfuerzo físico al que su fisiología de mujer no está ajustada.

Cuando hablan del principio de sorpresa también explicaban que una mujer en combate "ayudaría a aplicar el principio de sorpresa, dado que los GAO no tiene conocimiento de mujeres militares en la selva, y podrían pasar desapercibidas si toman apariencia de una compañera de ellos" (entrevistada 7).

En la última categoría, desarraigo de la vida civil, las entrevistadas consideran que lo que más extrañan de su vida civil es tener el control de su tiempo y hacer actividades que antes hacían como mujeres civiles. Tal como lo dice la entrevistada 6:

Cuando ingresas al Ejército, tomas otro modelo de mujer, ya no puedes ser esa niña mimada que puede hacer lo que quiera cuando lo planee, sino que tienes que ser una mujer fuerte, cumplir órdenes así te gusten o no, porque eres su comandante y tienes vidas a tu mando. Ya no respondes por tus acciones, sino que llevas en tus hombros la responsabilidad del mando, dejas de un segundo plano tus necesidades y lo único que importa son tus subalternos.

El comentario anterior deja ver nuevamente los estereotipos sociales de género por los que se encamina a la mujer a las actividades estéticas y a los hombres a las actividades físicas (Frömel et al., 2002).

\section{Discusión}

Tanto en Colombia como en Norteamérica existen políticas de inclusión. Por una parte, en Colombia existe el Decreto 2129 del 7 de octubre de 1976, que históricamente le permitió el ingreso a doce mujeres profesionales a las filas armadas, cuyo servicio siempre fue voluntario, con excepción de circunstancias en las que el país y el gobierno nacional exigieran lo contrario.

Del mismo modo, Estados Unidos desde 1910 creó el cuerpo de enfermeras del Ejército para cumplir con labores netamente logísticas. La gran diferencia entre 
los dos países es que en abril del 2016 el expresidente Barack Obama abrió todas las posiciones militares para las mujeres, incluidas las unidades de combate, mientras que en Colombia no se ha establecido ninguna política que las incluya directamente en posiciones de combate.

Los ejércitos de Colombia y de Estados Unidos cuentan con dos escuelas de formación, una de oficiales y otra de suboficiales. Ambos países desarrollan cursos de combate en tierra, aire y mar, de los cuales, la mujer militar colombiana en combate solo puede realizar el Curso Avanzado de Combate (CAC), y su participación está restringida en cursos como el de Lancero, Kaibil y Ranger; mientras que en Estados Unidos ya pueden hacer parte del cuerpo de Marines, Fuerzas Especiales y cursos de Ranger.

Al hacer un análisis reflexivo sobre la igualdad de género en ambos ejércitos, se evidencia que en Colombia existen menos posibilidades para la mujer que en Estados Unidos. Las sociedades son diferentes, pero la comparación tiene por objeto pensar en la incursión de la mujer militar dentro de una cultura estereotipada y con arraigo de identidades sociales establecidas por milenios desde lo masculino (el hombre como guerrero). La predominancia de lo masculino en el ejército no es un tema de solo países como Colombia, también lo vive la sociedad europea, el Oriente medio, Asia y EE. UU.; sin embargo, la movilización de la mujer hacia la incursión dentro del mundo militar ha sido un fenómeno desde la antigüedad. En consecuencia, los esfuerzos que se hacen ahora por legitimar su labor comprenden el fruto de años de incorporación y evolución de la presencia femenina en las filas. Si se observa en las entrevistas realizadas a las diez mujeres, hay una clara inclinación al servicio, factor que es de suma importancia para el desempeńo de cualquier actividad dentro del medio militar: la vocación resulta ser el llamado que la mayoría de las mujeres siente para dejar los estereotipos sociales de género que aún cobijan la sociedad actual.

La mujer en el Ejército no solo debe dejar su papel como civil, sino incorporarse en una cultura masculina, lo cual demanda un proceso de homogenización para lograr un encuadre social y adaptarse al medio militar. Por tanto, las mujeres militares se enfrentan a disyuntivas como la de caracterizarse con cualidades masculinas sin perder las femeninas, lo que implica una mayor exigencia emocional para ellas si quieren lograr una completa adaptación (Fernández \& Latorre, 2018).

Actualmente, en Colombia las mujeres tienen la posibilidad de escoger, desde su escuela de formación, entre siete armas, tres de las cuales son de combate, mientras que las otras cuatro son de apoyo para el combate. Por su parte, Estados Unidos cuenta con nueve armas y trece diferentes especialidades. Hay que tener en cuenta 
que, en Colombia, ningún militar puede escoger desde su escuela de formación el arma de Fuerzas Especiales.

Teniendo en cuenta los resultados del estudio y contrastándolos con los manuales de operaciones, se puede decir que la mujer combatiente tendría una mejor adaptabilidad en las Fuerzas Especiales, dado que el tiempo de operación es de quince días a un mes dependiendo de las implicaciones de la operación. El Ejército norteamericano no está haciendo control territorial como otra unidad normal que asegura su jurisdicción con estar de cuatro a cinco meses internos en la selva, sino que si la misión es de acción directa, ya tienen un objetivo claramente identificado, darían el golpe y se efectuaría su extracción del lugar. Si, por el contrario, no cuentan con información, la misión sería de reconocimiento, entonces, sí podrían durar más días en el área, el equipamiento sería más ligero, la ración, de menor cantidad.

Los soldados que conforman este grupo especial son soldados profesionales, suboficiales y oficiales que han luchado por llegar hasta ahí, no están prestando servicio militar obligatorio, por ende, su disposición es total a la disciplina de un militar. Asegura un coronel de Fuerzas Especiales que muchas veces los hombres pertenecientes a estos grupos se caracterizaron como mujeres para pasar desapercibidos por el enemigo, utilizando pelucas y elementos propios de una mujer. Esta actividad la podría hacer mejor una mujer, que no necesita de elementos que la ayuden a tomar esta figura.

En este sentido, la figura estereotipada de una mujer en el teatro de operaciones serviría para llegar más fácilmente a la población civil, prestar asistencia a las personas más vulnerables como niños y ancianos y promover cohesión en unidades pequeñas, tal y como lo demuestran los estudios de por Szayna et al. (2016).

\section{Conclusiones}

El análisis reflexivo sobre el papel de las mujeres en el Ejército a partir de la comparación de experiencias del Ejército de Estados Unidos y el Ejército de Colombia se convierte en un paso para seguir acercándose al reconocimiento de la labor de las mujeres militares en Colombia. Esto implica que se deben realizar más estudios comparativos de experiencias y buenas prácticas de otros países en cuanto a la incorporación del enfoque de género en sus ejércitos, como es el caso de Francia, España y, a nivel regional, Chile. Estos ejércitos vienen trabajando en equidad e igualdad entre hombres y mujeres dentro de sus procesos administrativos y operacionales de la mano de entidades como ONU Mujeres. 
Por otro lado, este estudio deja ver la necesidad de platear lineamientos estratégicos que profundicen en el perfil de las mujeres militares, teniendo en cuenta un enfoque de inclusión que ya viene adoptando el Ejército colombiano en materia de salud física y mental. Valdría la pena tener en cuenta dentro de estas disposiciones la labor de la mujer en el campo de combate, con el fin de generar rutas para garantizar sus condiciones de salubridad, protección de derechos y condiciones que el ejercicio del trabajo lo requiera, con el fin de recibir un trato equitativo e igualitario.

Finalmente, se aborda la necesidad de la transversalización del enfoque de género para lograr que todos los procesos, procedimientos y acciones dentro de la institución apunten al fomento de la cultura de equidad. Para ello, se deben tener en cuenta los contextos sociales en los que se habita y tratar de romper con los estereotipos sociales de género que limitan la labor de las mujeres militares.

\section{Referencias}

Antolín, L. (2003). La mitad invisible. Género en la educación para el desarrollo. Ecsur.

Asch, B. J., Miller, T., \& Weinberger, G. (2016). Can we explain gender differences in officer career progression? RAND Corporation.

Brailovsky, D., Herrera, S., \& Santa Cruz, E. (2001). La desigualdad en la educación para varones y mujeres en el jardin de infantes. En 0 a 5 la educación en los primeros años. Centro de Publicaciones Educativas y Material Didáctico S.R.L.

Brown, L., \& Soto, H. (2007). Reflexiones sobre equidad de género y educación inicial. InterSedes. Revista de las Sedes Regionales, 8(14), 33-48.

Caicedo, A., García, M., López, E., Castillo, A., Narváez, A., Ramírez, H., Barreto, J., Lucero, J., \& Londońo, J. D. (2017). Mujeres militares. Historias de grandeza al servicio de la paz. ONU mujeres; Comando General de las Fuerzas Militares de Colombia.

Canto, J., \& Moral, F. (2005). El sí mismo desde la teoría de la identidad social. Escritos de Psicología, 7, 59-70.

Devereux, G. (2014). Capital controls, global liquidity traps, and the international policy trilemma. The Scandinavian Journal of Economics, 116, 158-189.

Dos mujeres hacen historia militar en EE. UU. al convertirse en las primeras 'Ranger'. (2015). La Información. https://www.lainformacion.com/mundo/dos-mujeres-hacen-historia-militar-en-eeuu-al-convertirse-en-las-primeras-ranger_wgtpu8whhce0bokwsaclj4/

Echevarría, C. (1990). Aproximación psicosociológica. La formación militar y sus roles, pautas, escenarios y objetivos futuros. Cuaderno de Estrategia, 19, 59-81.

Fernández, A., \& Latorre E. J. (Eds.). (2018). La construcción del rol de la mujer militar. Escuela Militar de Cadetes “General José María Córdova”. https://doi.org/10.21830/9789585692831

Fernández, A., \& Latorre, E. J. (2018). Perfil sociodemográfico de los futuros oficiales del Ejército Nacional de Colombia. En A. Fernández, \& E. J. Latorre (Eds.), La construcción del rol de 
la mujer militar. Escuela Militar de Cadetes "General José María Córdova”. https://doi. org/10.21830/9789585692831

Frömel, K., Formánková, S., \& Sallis, J. F. (2002). Physical activity and sport preferences of 10 to 14 year-old children: A 5-year prospective study. Acta Universitatis Palackianae Olomucensis. Gymnica, 32(1), 11-16.

Gallardo, P. (2012). La mujer militar en las Fuerzas Armadas. Cuadernos de Estrategia, 157, 139-174.

González, A. (2005). Aspectos psicosociales de la integración de la mujer en las Fuerzas Armadas. En Mujer, Fuerzas Armadas y conflictos bélicos: una visión panorámica. Ministerio de Defensa.

Herrera Gómez, M. (2000). La relación social como categoría de las ciencias sociales. REIS, Revista Española de Investigaciones Sociológicas, 90, 37-77. https://www.redalyc.org/articulo. oa?id $=99717877002$

Kimble, C., Hirt, E., Díaz-Loving, R., Hosch, H., Lucker, G. W., \& Zárate, M. (2002). Psicología social de las Américas. Pearson.

Palomar Verea, Cristina. (2005). La política de género en la educación superior. La Ventana. Revista de Estudios de Género, 3(21), 7-43. http://www.scielo.org.mx/scielo.php?script=sci_arttext\&pi$\mathrm{d}=$ S1405-94362005000100007\&lng=es\&tlng=es

Phillips, D. (2017). Mujeres en combate: el valor femenino en la línea de fuego. The New York Times. https://www.nytimes.com/es/2017/06/10/espanol/mujeres-a-combate-el-valor-femenino-enla-linea-de-fuego.html

Schaefer, A. G., Wenger, J., Kavanagh, J., Wong, J., Oak, G., Trail, T., \& Nichols, T. (2015). Implications of integrating women into the Marine Corps Infantry. RAND Corporation.

Szayna, T. S., Larson, E., O’Mahony, A., Robson, S., Schaefer, A., Matthews, M., Michael Polich, J. M., Ayer, L., Eaton, D., Marcellino, W., Kraus, L., Posard, M., Syme, J., Winkelman, Z., Wright, C., Zander, M., \& Welser. W. (2016). Considerations for integrating women into closed occupations in U. S. Special Operations Forces. RAND Corporation.

Tajfel, H. (1978). Differentiation between social groups: Studies in the social psychology of intergroups relations. Londres: Academic Press.

Taylor, S. J., \& Bogdan, R. (1998). Introduction to qualitative research methods: A guide and resource (3a Ed.). John Wiley \& Sons. 